\title{
Sexual orientation and quality of life among students of Obafemi Awolowo University (OAU), Nigeria.
}

\author{
Mapayi Boladale ${ }^{1,2}$, Oginni Olakunle ${ }^{2}$, Aloba Olutayo ${ }^{1,2}$, Akinsulore Adesanmi ${ }^{1,2}$
}

1. Obafemi Awolowo University, Ile-Ife, Nigeria

2. Obafemi Awolowo University Teaching Hospitals Complex, Ile-Ife, Nigeria

\begin{abstract}
Background: Sexual orientation is an individual's pattern of physical and emotional arousal toward members of the same and/or opposite gender.

Objective: To determine the pattern of sexual orientation and the relationship between sexual orientation and quality of life among a sample of OAU students.

Methods: A descriptive cross sectional study among 481 students of OAU using a multistage sampling technique. They completed a Socio-demographic data schedule, questions on sexual orientation and the World Health Organization Quality of Life Scale - Brief version (WHO QOL-BREF).

Results: $4.9 \%$ of the sample self-identified as bisexual while $0.1 \%$ self-identified as gay/lesbian. $11.8 \%$ of the respondents reported varying degrees of attraction to the opposite gender. The mean age of sexual debut was $17.62( \pm 4.05)$. Those who self-identified as gay/lesbian/bisexual had a lower average score on all domains of the WHO QOL-BREF.

Conclusion: Same sex sexual attraction and practice occur among young people in Nigeria and this has sexual and reproductive health implications. GLB youth report a lower QOL compared to heterosexual counterparts and this may suggest some distress among this vulnerable group. More studies should be undertaken to explore issues raised in planning interventions and health services that would improve safe sexual practices within this group.
\end{abstract}

Keywords: Sexual orientation, quality of life, students, Nigeria.

DOI: http://dx.doi.org/10.4314/ahs.v15i4.3

Cite as: Boladale M, Olakunle O, Olutayo A, Adesanmi A. Sexual orientation and quality of life among students of Obafemi Awolowo University (OAU), Nigeria. Afri Health Sci. 2015;15(4):1065-73. bttp:// dx.doi.org/10.4314/abs.v15i4.3

\section{Introduction}

Sexual orientation is an individual's pattern of physical and emotional arousal toward members of the same and/or opposite gender ${ }^{1}$. It exists along a continuum that ranges from exclusive heterosexuality to exclusive homosexuality and includes various degrees of bisexuality. Sexual orientation is different from sexual behavior because it refers to feelings and self-concept. Individuals may or may not express their sexual orientation in their behaviors ${ }^{2,3}$. Conceptually, sexual orientation

\author{
Corresponding author: \\ Mapayi Boladale, \\ Obafemi Awolowo University \\ Teaching Hospitals Complex, \\ Ile-Ife, Nigeria \\ Mapayi Boladale: \\ Tel: +2348033930096, \\ daledosu@yahoo.com
}

has three major dimensions, Sexual attraction, which is the sex or gender of individuals that someone feels attracted to ${ }^{4}$; sexual behavior, which is the sex of sex partners ${ }^{5}$ and self-identification, which is how one identifies one's sexual orientation. Sexual attraction is the main construct included in definitions of sexual orientation and it is important for young people and others who are not sexually active ${ }^{5}$. Authors have posited that sexual attraction is the most important construct for measuring sexual orientation ${ }^{6,7}$.

Not everyone with same-sex attraction engage in sexual activity with partners of the same $\operatorname{sex}^{5,8}$ and vice versa. It is thus important to know if there are discrepancies in attraction and behaviour. Self-identification varies over time for most individuals and is heavily influenced by socio- cultural factors ${ }^{3}$. Self-identification is not always in agreement with sexual behavior or attraction ${ }^{5,9}$. The discordance among these three dimensions are likely due to multiple factors and these include stigma, laws and legal risks in some countries, especially in a country like Nigeria where same sex relationships 
are criminalized, cultural values and meanings, developmental change, partner selection opportunities and even economic considerations ${ }^{3}$. Moreover, the factors could also be operational and methodological, for example discrepancies in sampling, instruments used and measurement errors. The complex components of sexual orientation challenge many adolescents' emotional and psychological development ${ }^{10}$. Studies in Cuba, Norway, India, and South Africa have shown that sexual orientation affects quality-of-life measures ${ }^{11,12}$.

Quality Of Life (QOL) is a highly complex concept. Although there is no universal definition for QOL, the construct usually includes subjective well-being and objective mental, physical and social functioning indicators $^{13,14}$. QOL has been defined as an individual's sense of well-being and satisfaction with his or her life circumstances, as well as his or her health status and access to resources and opportunities ${ }^{15}$. The World Health Organization (WHO) defined QOL as the "individual's perception of his or her position in life, within the cultural context and value system he or she lives in, and in relation to his or her goals, expectations, parameters and social relations. It is a far reaching concept, affected by the persons' physical health, psychological state, level of independence, social relationships and their relationship to salient features of their environment" through complex mechanisms ${ }^{16}$.

\section{Objective}

The objective of this study was to determine the prevalence of the different patterns of sexual orientation and the relationship between sexual orientation and quality of life among a sample of OAU students.

\section{Methods}

The study was of descriptive cross sectional design. The student population of Obafemi Awolowo University Ile-Ife Osun state, situated in the South Western geographical zone of Nigeria constituted the population from which a sample was drawn. A multistage, stratified sampling technique was used to recruit students for the study. There are 10 halls of residence. There are four male halls, four female halls, one postgraduate hall and one other female hall located within the university campus but managed by a private organisation.

The sample size that was used for this study was computed based on the formula by Lenth ${ }^{17}$ and Araoye ${ }^{18}$. Since there was a dearth of studies on sexual orientation in Nigeria and there are great disparities in global figures depending on the region being investigated, the figure of $50 \%$ suggesting that the prevalence is largely unknown was employed. A sample size of 384 was arrived at as the minimum but a sample size of 500 was used to increase the power of the study and account for instances of drop outs and incomplete data.

A multistage, stratified, systematic sampling technique was used in this study.

Stage 1: All the halls of residence were stratified into 2 based on gender. In total, 4 male halls and 4 female halls were included in the study.

Stage 2: The total number of students and total number of rooms in each of the selected halls was obtained from the hall supervisors of the halls. Five hundred questionnaires were distributed among the 8 halls. The number to be interviewed in each hall was determined by proportional sampling method.

Stage 3: After determining the number to be interviewed in each hall, respondents were chosen by systematic random sampling. Odd numbered rooms on odd numbered floors were selected and then one student was randomly selected from each odd numbered room until the target study number for that hall was achieved.

The research instruments included a semi structured Socio-demographic data schedule, the World Health Organization Quality of Life Scale - Brief version (WHO QOL-BREF) and questions on sexual orientation. A semi structured Socio-demographic data schedule was designed purposely for this study to elicit information on variables such as age, gender, level of study, marital status of parents, ethnicity, religion and questions on sexual practices. Three sexual orientation questions were added as recommended by the SMART Collaboration of the Williams Institute ${ }^{3}$ to include the constructs of sexual identity, attraction and behaviour. The WHO QOL- BREF is a 26-item self-administered generic questionnaire. It is a short version of the WHO QOL -100 scales $^{16}$. The WHO QOL - BREF is an international quality of life instrument which produces a profile with four domain scores: physical health (7 items), psychological health (6 items), social relationships (3 items), environmental domain (8 items) as well as two separately scored items about the individuals' perception of their quality of life (QI) and health (Q2). Each item is scored in a Likert format from 1 to 5 . The WHO QOL - BREF has been validated across a wide variety of cultures, including Nigeria ${ }^{19}$.

Data analysis was carried out using the SPSS 16 soft- 
ware. To achieve the objectives of the study, appropriate descriptive and inferential statistics were used for the data collected. Univariate analysis was used to determine the prevalence of different forms of sexual orientation and these were expressed in percentages. Association at bivariate level was assessed using student $t$ test and chi square test. A p value of $<0.05$ was considered statistically significant in all cases.

The study protocol was presented for approval to the Research and Ethical Committee of the Institute of Public Health, Obafemi Awolowo University. The nature of the study, its aims and objectives were explained to the participants and written consent freely obtained. The participants were assured of confidentiality.

\section{Results}

Five hundred questionnaires were administered and 481 questionnaires were included in the final analysis giving a response rate of $96.2 \%$.

\section{Socio-demographic profile}

The socio-demographic profile of participants was disaggregated by gender and is presented on Table 1 . Most of the respondents were aged 21-25 years (50.6\%), with more of the female respondents being in younger age groups $\left(\chi^{2}=28.622, \mathrm{p}<0.001\right)$. Most of the respondents $(85.1 \%)$ were Yoruba. Ten $(2.1 \%)$ of the respondents were married, most of whom were females $(80 \%)$. Majority $(87.4 \%)$ of the respondents were christians and the median monthly allowance for most was N12,000 (Interquartile range N10,000). Both male and female respondents were comparable with regard to other socio-demographic variables.

Table 1: Socio-demographic characteristics of respondents

\begin{tabular}{|c|c|c|c|c|c|c|}
\hline \multirow{2}{*}{\multicolumn{2}{|c|}{ Socio-demographics }} & \multicolumn{3}{|l|}{ Gender } & \multirow[b]{2}{*}{$\chi^{2}$} & \multirow[b]{2}{*}{$\mathbf{p}$} \\
\hline & & $\begin{array}{l}\text { Male } \\
\text { n (\%) }\end{array}$ & $\begin{array}{l}\text { Female } \\
\text { n }(\%)\end{array}$ & $\begin{array}{l}\text { Total } \\
\text { n (\%) }\end{array}$ & & \\
\hline \multirow{4}{*}{$\begin{array}{l}\text { Age } \\
\text { (years) } \\
(n=480)\end{array}$} & $\leq \mathbf{2 0}$ & $43(20.7)$ & $89(32.7)$ & $\begin{array}{l}132 \\
(27.5)\end{array}$ & \multirow[t]{4}{*}{28.622} & \multirow[t]{4}{*}{$<0.001$} \\
\hline & $21-25$ & $105(50.5)$ & $138(50.7)$ & $\begin{array}{l}243 \\
(50.6)\end{array}$ & & \\
\hline & $26-30$ & $41(19.7)$ & $14(5.1)$ & $55(11.5)$ & & \\
\hline & $>\mathbf{3 0}$ & $19(9.1)$ & $31(11.4)$ & $50(10.4)$ & & \\
\hline \multirow{4}{*}{$\begin{array}{l}\text { Ethnicity } \\
(n=477)\end{array}$} & Yoruba & $171(82.6)$ & $235(87.0)$ & $\begin{array}{l}406 \\
(85.1)\end{array}$ & \multirow[t]{4}{*}{2.931} & \multirow[t]{4}{*}{0.402} \\
\hline & Igbo & $18(8.7)$ & $17(6.3)$ & $35(7.3)$ & & \\
\hline & Edo/Delta & $13(6.3)$ & $10(3.7)$ & $23(4.8)$ & & \\
\hline & Others & $5(2.4)$ & $8(3.0)$ & $13(2.7)$ & & \\
\hline \multirow{2}{*}{$\begin{array}{l}\text { Marital } \\
\text { Status } \\
(n=475)\end{array}$} & Single & $203(99.0)$ & $262(97.0)$ & $\begin{array}{l}465 \\
(97.9)\end{array}$ & \multirow[t]{2}{*}{2.233} & \multirow[t]{2}{*}{0.199} \\
\hline & Married & $2(1.0)$ & $8(3.0)$ & $10(2.1)$ & & \\
\hline \multirow{3}{*}{$\begin{array}{l}\text { Religion } \\
(n=468)\end{array}$} & Christianity & $171(85.9)$ & $238(88.5)$ & $\begin{array}{l}409 \\
(87.9)\end{array}$ & \multirow[t]{3}{*}{1.610} & \multirow[t]{3}{*}{0.47} \\
\hline & Echist & $0(0.0)$ & $1(0.4)$ & $1(0.2)$ & & \\
\hline & Islam & $28(14.1)$ & $30(11.2)$ & $58(12.4)$ & & \\
\hline \multirow{2}{*}{$\begin{array}{l}\text { Level of } \\
\text { study } \\
(n=468)\end{array}$} & Undergraduate & $198(97.5)$ & 264 (99.9) & $\begin{array}{l}462 \\
(98.7)\end{array}$ & \multirow[t]{2}{*}{3.951} & \multirow[t]{2}{*}{0.09} \\
\hline & Postgraduate & $5(2.5)$ & $1(0.4)$ & $6(1.3)$ & & \\
\hline \multirow{3}{*}{$\begin{array}{l}\text { Monthly } \\
\text { allowance } \\
(\mathbf{N}, \mathbf{0 0 0}) \\
(\mathbf{n}=\mathbf{4 8 0})\end{array}$} & $<10$ & $134(64.7)$ & $177(64.8)$ & $\begin{array}{l}311 \\
(64.8)\end{array}$ & \multirow[t]{3}{*}{1.567} & \multirow[t]{3}{*}{0.457} \\
\hline & $10-20$ & $50(24.2)$ & $74(27.1)$ & $\begin{array}{l}124 \\
(25.8)\end{array}$ & & \\
\hline & $>\mathbf{2 0}$ & $23(11.1)$ & $22(8.1)$ & $45(9.4)$ & & \\
\hline
\end{tabular}




\section{Sexual behaviour}

Table 2 shows the sexual behaviour of respondents disaggregated by gender. Fifty three $(11.8 \%)$ of the respondents reported varying degrees of attraction to the opposite gender with female respondents being more likely to report varying degrees of attraction to the same gender $(\chi 2=29.473, \mathrm{p}<0.001)$. With respect to actual sexual behaviour, more female respondents denied having had sex, but were also more likely to report same gender sexual activity $\left(\chi^{2}=2.123, \mathrm{p}=0.017\right)$. Twenty five $(5.6 \%)$ of the respondents reported their sexual orientation as gay $(0.7 \%)$ or bisexual $(4.9 \%)$. The mean age of sexual debut was 17.62 ( \pm 4.05$)$, with female respondents being more likely to report an older age of sexual debut $\left(\chi^{2}=13.940, p=0.003\right)$ compared with the males.

Table 2: Sexual behaviour of respondents

\begin{tabular}{|c|c|c|c|c|c|c|}
\hline \multirow{2}{*}{\multicolumn{2}{|c|}{ Sexual Behaviour }} & \multicolumn{3}{|c|}{ Gender } & \multirow[b]{2}{*}{$\chi^{2}$} & \multirow[b]{2}{*}{$\mathbf{p}$} \\
\hline & & $\begin{array}{l}\text { Male } \\
\text { n (\%) }\end{array}$ & $\begin{array}{c}\text { Female } \\
\text { n }(\%)\end{array}$ & $\begin{array}{l}\text { Total } \\
\text { n (\%) }\end{array}$ & & \\
\hline \multirow{5}{*}{$\begin{array}{l}\text { Sexual } \\
\text { attraction } \\
(n=448)\end{array}$} & $\begin{array}{l}\text { Exclusively } \\
\text { opposite sex }\end{array}$ & $155(77.9)$ & $140(56.2)$ & $295(65.8)$ & 29.473 & $<0.001$ \\
\hline & $\begin{array}{l}\text { Predominantly } \\
\text { opposite sex }\end{array}$ & $36(18.1)$ & $64(25.7)$ & $100(22.3)$ & & \\
\hline & $\begin{array}{l}\text { Equally both } \\
\text { sexes }\end{array}$ & $7(3.5)$ & $41(16.5)$ & $48(10.7)$ & & \\
\hline & $\begin{array}{l}\text { Predominantly } \\
\text { same sex }\end{array}$ & $1(0.5)$ & $3(1.2)$ & $4(0.9)$ & & \\
\hline & $\begin{array}{l}\text { Exclusively } \\
\text { same sex }\end{array}$ & $0(0.0)$ & $1(0.4)$ & $1(0.2)$ & & \\
\hline \multirow{4}{*}{$\begin{array}{l}\text { Sexual } \\
\text { behavior } \\
(n=469)\end{array}$} & Opposite sex & $87(42.2)$ & $79(30.0)$ & $166(35.4)$ & 9.123 & 0.017 \\
\hline & Both sexes & $2(1.0)$ & $5(1.9)$ & $7(1.5)$ & & \\
\hline & Same sex & $0(0)$ & $2(0.8)$ & $2(0.4)$ & & \\
\hline & Missing & $117(56.8)$ & $177(67.3)$ & $294(62.7)$ & & \\
\hline \multirow{3}{*}{$\begin{array}{l}\text { Sexual } \\
\text { orientation } \\
(n=452)\end{array}$} & Heterosexual & $189(95.5)$ & $238(93.7)$ & $427(94.5)$ & 0.655 & 0.418 \\
\hline & Bisexual & $8(4.0)$ & $14(5.5)$ & $22(4.9)$ & & \\
\hline & Gay & $1(0.5)$ & $2(0.8)$ & $3(0.7)$ & & \\
\hline \multirow{2}{*}{$\begin{array}{l}\text { Gender of } \\
\text { first partner } \\
(\mathrm{n}=\mathbf{2 0 8})\end{array}$} & $\begin{array}{l}\text { Opposite } \\
\text { Gender }\end{array}$ & $106(95.5)$ & $90(92.8)$ & $196(94.2)$ & 0.700 & 0.403 \\
\hline & Same sex & $5(4.5)$ & $7(7.2)$ & $12(5.8)$ & & \\
\hline \multirow{4}{*}{$\begin{array}{l}\text { Age at sexual } \\
\text { debut }(n=481)\end{array}$} & $\leq 10$ & $6(2.9)$ & $6(2.2)$ & $12(2.5)$ & 13.940 & 0.003 \\
\hline & 11.15 & $16(7.7)$ & $12(4.4)$ & $28(5.8)$ & & \\
\hline & $16-20$ & $62(29.8)$ & 49 (17.9) & $111(23.1)$ & & \\
\hline & $\geq 21$ & $124(59.6)$ & $206(75.5)$ & $330(68.6)$ & & \\
\hline \multirow{2}{*}{$\begin{array}{l}\text { Dream of sex } \\
(n=260)\end{array}$} & Yes & $25(16.4)$ & $16(14.8)$ & $41(15.8)$ & 0.127 & 0.722 \\
\hline & No & $127(83.6)$ & $92(85.2)$ & $219(84.2)$ & & \\
\hline \multirow{2}{*}{$\begin{array}{l}\text { Masturbate } \\
(n=203)\end{array}$} & Yes & 21(19.4) & 17 (17.9) & 38 (18.7) & 0.080 & 0.778 \\
\hline & Rarely/Never & $87(80.6)$ & $78(82.1)$ & $165(83.1)$ & & \\
\hline \multirow{2}{*}{$\begin{array}{l}\text { Protection } \\
\text { during sex } \\
(\mathrm{n}=236)\end{array}$} & Yes & $95(74.8)$ & $64(58.7)$ & $159(67.4)$ & 6.906 & 0.009 \\
\hline & No & $32(25.2)$ & $45(41.3)$ & $77(32.6)$ & & \\
\hline \multirow{2}{*}{$\begin{array}{l}\text { Currently } \\
\text { sexually active } \\
(n=389)\end{array}$} & Yes & $112(60.2)$ & $72(35.5)$ & $184(47.3)$ & 23.847 & $<0.001$ \\
\hline & No & $74(39.8)$ & $131(64.5)$ & $205(52.7)$ & & \\
\hline \multirow{2}{*}{$\begin{array}{l}\text { Penovaginal } \\
\operatorname{sex}(n=478)\end{array}$} & Yes & $89(43.2)$ & $67(24.6)$ & $156(32.6)$ & 18.390 & $<0.001$ \\
\hline & No & $117(56.8)$ & $205(75.4)$ & $322(67.4)$ & & \\
\hline \multirow{2}{*}{$\begin{array}{l}\text { Anal sex } \\
(n=479)\end{array}$} & Yes & $22(10.6)$ & $20(7.4)$ & $42(8.8)$ & 1.576 & 0.209 \\
\hline & No & $185(89.4)$ & $252(92.6)$ & $437(91.2)$ & & \\
\hline \multirow{2}{*}{$\begin{array}{l}\text { Oral sex } \\
(n=479)\end{array}$} & Yes & $47(22.7)$ & $31(11.4)$ & $78(16.3)$ & 11.026 & 0.001 \\
\hline & No & $160(77.3)$ & $241(88.6)$ & $401(83.7)$ & & \\
\hline
\end{tabular}


Forty one $(8.5 \%)$ of the respondents dreamt about sex, while $38(18.7 \%)$ masturbated regularly. One hundred and fifty nine $(67.4 \%)$ used protection during sex and about a third $(38.3 \%)$ reported being sexually active. A third (32.6\%) practiced penovaginal sex, while 42 $(8.8 \%)$ practiced anal sex and $78(16.3 \%)$ practiced oral sex. Male respondents were significantly more likely to use protection while having sex, as well as to report being currently sexually active. They were also significantly more likely to report engaging in masturbation, penovaginal, anal and oral sex.

Respondents who identified as gay/bisexual were significantly more likely to report dreaming of sex, masturbating regularly, being sexually active and practising oral and anal sex (Table 3). Although even in heterosexual relationships, $7.5 \%$ practiced anal sex.

Table 3: Association between sexual orientation and sexual behaviour of respondents

\begin{tabular}{|c|c|c|c|c|c|c|}
\hline \multirow{2}{*}{\multicolumn{2}{|c|}{ Sexual Behaviour }} & \multicolumn{3}{|c|}{ Sexual orientation } & \multirow{4}{*}{$\begin{array}{c}\chi^{2} \\
18.449\end{array}$} & \multirow{4}{*}{$\begin{array}{l}\text { p } \\
<0.001\end{array}$} \\
\hline & & \multirow{2}{*}{$\begin{array}{c}\begin{array}{c}\text { Heterosexual } \\
\text { n (\%) }\end{array} \\
30(13.2)\end{array}$} & \multirow{2}{*}{$\begin{array}{c}\text { Gay/bisexual } \\
\text { n (\%) } \\
10(50.0)\end{array}$} & \multirow{2}{*}{$\begin{array}{l}\begin{array}{l}\text { Total } \\
\text { n (\%) }\end{array} \\
40(16.1)\end{array}$} & & \\
\hline Dream of & Yes & & & & & \\
\hline $\operatorname{sex}(n=248)$ & No & $198(86.8)$ & $10(50.0)$ & $208(83.9)$ & & \\
\hline \multirow{2}{*}{$\begin{array}{l}\text { Masturbate } \\
(n=195)\end{array}$} & Yes & $29(16.5)$ & $9(47.4)$ & $38(19.5)$ & 10.430 & 0.003 \\
\hline & Rarely/Never & $147(83.5)$ & $10(52.6)$ & $157(80.5)$ & & \\
\hline \multirow{2}{*}{$\begin{array}{l}\text { Currently } \\
\text { sexually } \\
\text { active } \\
(\mathrm{n}=369) \\
\end{array}$} & Yes & $161(46.4)$ & $15(68.2)$ & $176(47.7)$ & 3.935 & 0.047 \\
\hline & No & $186(53.6)$ & $7(31.8)$ & $193(52.3)$ & & \\
\hline \multirow{2}{*}{$\begin{array}{l}\begin{array}{l}\text { Protection } \\
\text { during sex } \\
(n=224)\end{array} \\
\end{array}$} & Yes & $141(68.8)$ & $12(63.2)$ & $153(68.3)$ & 0.254 & 0.614 \\
\hline & No & $64(31.2)$ & $7(36.8)$ & $71(31.7)$ & & \\
\hline \multirow{2}{*}{$\begin{array}{l}\text { Penovaginal } \\
\operatorname{sex}(n=450)\end{array}$} & Yes & $146(34.4)$ & $9(36.0)$ & $155(34.4)$ & 0.028 & 0.866 \\
\hline & No & $279(65.6)$ & $16(64.0)$ & $295(65.6)$ & & \\
\hline \multirow{2}{*}{$\begin{array}{l}\text { Anal sex } \\
(n=450)\end{array}$} & Yes & $32(7.5)$ & $9(36.0)$ & $41(9.1)$ & 23.111 & $<0.001$ \\
\hline & No & $393(92.5)$ & $16(64.0)$ & $409(90.9)$ & & \\
\hline \multirow{2}{*}{$\begin{array}{l}\text { Oral sex } \\
(n=450)\end{array}$} & Yes & $65(15.3)$ & $11(44.0)$ & $76(16.9)$ & 13.861 & 0.001 \\
\hline & No & $360(84.7)$ & $14(56.0)$ & $374(83.1)$ & & \\
\hline
\end{tabular}

\section{Quality of life}

Table 4 shows the variables associated with overall quality of life and health satisfaction. Female respondents were more likely to reported good overall quality of life $\left(\chi^{2}=13.940, p=0.003\right)$ while older respondents reported higher overall quality of life and health satisfaction $\left(\chi^{2}=13.940, p=0.003\right)$. Respondents who worshipped regularly also reported higher overall quality of life $(\chi 2=13.940, p=0.003)$. Younger students reported poorer physical health, compared with older students $\left(\chi^{2}=13.940, \mathrm{p}=0.003\right)$. 
Table 4: Variables associated with Overall Quality of Life and Health Satisfaction

\begin{tabular}{|c|c|c|c|c|c|c|c|c|c|c|}
\hline \multirow{2}{*}{\multicolumn{2}{|c|}{ Socio-demographics }} & \multicolumn{4}{|c|}{ Overall QOL } & \multicolumn{5}{|c|}{ Health Satisfaction } \\
\hline & & $\begin{array}{l}\text { Fair } \\
\text { n (\%) }\end{array}$ & $\begin{array}{l}\text { Good } \\
\text { n (\%) }\end{array}$ & $\chi^{2}$ & p & $\begin{array}{l}\text { Poor } \\
\text { n (\%) }\end{array}$ & $\begin{array}{l}\text { Fair } \\
\text { n (\%) }\end{array}$ & $\begin{array}{l}\text { Good } \\
\text { n (\%) }\end{array}$ & $\chi^{2}$ & $\mathbf{p}$ \\
\hline \multirow{2}{*}{ Sex } & Male & $147(49.3)$ & $61(34.3)$ & 10.272 & 0.001 & $1(50.0)$ & $145(45.5)$ & $62(39.7)$ & 1.423 & 0.644 \\
\hline & Female & $151(50.7)$ & $117(65.7)$ & & & $1(50.0)$ & $174(54.5)$ & $94(60.3)$ & & \\
\hline \multirow{4}{*}{ Age } & $\leq \mathbf{2 0}$ & $79(26.5)$ & $53(29.9)$ & 8.451 & $\mathbf{0 . 0 3 8}$ & $0(0.0)$ & $93(29.2)$ & $39(25.2)$ & 17.337 & 0.021 \\
\hline & $21-25$ & $161(54.0)$ & 79 (44.6) & & & $1(50.0)$ & $168(52.7)$ & $71(45.8)$ & & \\
\hline & $26-30$ & $36(12.1)$ & $19(10.7)$ & & & $0(0.0)$ & $37(11.6)$ & $18(11.6)$ & & \\
\hline & $>\mathbf{3 0}$ & $22(7.4)$ & $26(14.7)$ & & & $1(50.0)$ & $21(6.6)$ & $27(17.4)$ & & \\
\hline \multirow{2}{*}{$\begin{array}{l}\text { Marital } \\
\text { Status }\end{array}$} & Single & $290(98.3)$ & $170(97.1)$ & 0.712 & 0.511 & $2(100.0)$ & $312(98.4)$ & $147(96.7)$ & 1.493 & 0.334 \\
\hline & Married & $5(1.7)$ & $5(2.9)$ & & & $0(0.0)$ & $5(1.6)$ & $5(3.3)$ & & \\
\hline \multirow{3}{*}{ Income } & $<10$ & $187(63.0)$ & $119(66.9)$ & 1.113 & 0.573 & $2(100.0)$ & $198(62.3)$ & 107 (68.6) & 3.086 & 0.503 \\
\hline & $10-20$ & $79(26.6)$ & $45(25.3)$ & & & $0(0.0)$ & $87(27.4)$ & $37(23.7)$ & & \\
\hline & $>\mathbf{2 0}$ & $31(10.4)$ & $14(7.9)$ & & & $0(0.0)$ & $33(10.4)$ & $12(7.7)$ & & \\
\hline \multirow{3}{*}{$\begin{array}{l}\text { Regularity } \\
\text { of worship }\end{array}$} & Regularly & $218(79.3)$ & 147 (88.6) & 7.886 & 0.019 & $1(50.0)$ & $235(79.4)$ & $130(90.3)$ & 10.437 & 0.102 \\
\hline & Occassionally & $42(15.3)$ & $17(10.2)$ & & & $1(50.0)$ & 47 (15.9) & $11(7.6)$ & & \\
\hline & Rarely ever & $15(5.5)$ & $2(1.2)$ & & & $0(0.0)$ & $14(4.7)$ & $3(2.1)$ & & \\
\hline \multirow{2}{*}{$\begin{array}{l}\text { Level of } \\
\text { study }\end{array}$} & Undergraduate & $289(99.3)$ & $168(97.7)$ & 2.268 & 0.201 & $2(100.0)$ & 308 (99.7) & $148(96.7)$ & 6.977 & 0.042 \\
\hline & Postgraduate & $2(0.7)$ & $4(2.3)$ & & & $0(0.0)$ & $1(0.3)$ & $5(3.3)$ & & \\
\hline \multirow{2}{*}{$\begin{array}{l}\text { Sexual } \\
\text { orientation }\end{array}$} & Heterosexual & 264 (93.6) & $159(95.8)$ & 0.930 & 0.335 & $2(100.0)$ & $\begin{array}{r}286 \\
(94.7))\end{array}$ & $135(93.8)$ & 0.286 & 0.845 \\
\hline & $\begin{array}{l}\text { Gay and } \\
\text { bisexual }\end{array}$ & $18(6.4)$ & $7(4.2)$ & & & $0(0.0)$ & $16(5.3)$ & $9(6.3)$ & & \\
\hline
\end{tabular}

Table 5 showed the relationship between quality of life lower means in all components of QOL compared with and sexual orientation. Gay/bisexual students reported heterosexuals and this was statistically significant in all but one of the components (environment).

Table 5: Association between sexual orientation and Quality of Life

\begin{tabular}{|c|c|c|c|c|c|}
\hline $\begin{array}{l}\text { Quality of Life } \\
\text { Domains }\end{array}$ & $\begin{array}{c}\text { Sexual } \\
\text { Orientation }\end{array}$ & Mean & $\begin{array}{c}\text { Std. } \\
\text { Deviation }\end{array}$ & $\mathbf{F}$ & $\mathbf{p}$ \\
\hline \multirow[b]{2}{*}{ Physical Health } & Heterosexual & 15.20 & 2.06 & 8.701 & .003 \\
\hline & $\begin{array}{l}\text { Gay and } \\
\text { bisexual }\end{array}$ & 13.92 & 1.90 & & \\
\hline \multirow[b]{2}{*}{ Psychological domain } & Heterosexual & 16.14 & 1.93 & 6.286 & .013 \\
\hline & $\begin{array}{l}\text { Gay and } \\
\text { bisexual }\end{array}$ & 15.13 & 2.42 & & \\
\hline \multirow[b]{2}{*}{ Social relationships } & Heterosexual & 15.46 & 2.56 & 11.104 & .001 \\
\hline & $\begin{array}{l}\text { Gay and } \\
\text { bisexual }\end{array}$ & 13.71 & 2.56 & & \\
\hline \multirow[b]{2}{*}{ Environment } & Heterosexual & 13.80 & 2.23 & .415 & .520 \\
\hline & $\begin{array}{l}\text { Gay and } \\
\text { bisexual }\end{array}$ & 13.50 & 2.28 & & \\
\hline \multirow[b]{2}{*}{ Total score } & Heterosexual & 68.66 & 7.95 & 9.276 & .002 \\
\hline & $\begin{array}{l}\text { Gay and } \\
\text { bisexual }\end{array}$ & 63.70 & 7.04 & & \\
\hline
\end{tabular}




\section{Discussion}

\section{Sexual behaviour}

In this study, it is clear that as expected there appears to be some discrepancy between sexual identity, attraction and behaviour as stated by previous authors ${ }^{3,5,8,9}$. A lot of missing data under sexual practices may reflect unwillingness taboo associated with open discussion of sexual matters in this environment but it might also be that non responders are more likely to be gay/bisexuals who are afraid to self-identify because of the cultural, religious and legal implications ${ }^{20}$. Female respondents were more likely to report non-heterosexual activity. This finding is supported in literature. Ventegodt reported among a Danish sample that prevalence of both homosexuality and bisexuality was higher among female respondents compared to the males ${ }^{20}$. Reasons for this may be varied. Men in this environment may be less likely to report homosexuality. Homosexuality may be seen as non-masculine and thus a threat to a man's masculinity.

The proportion of respondents who self-identified as gay/bisexual in our study is comparable to reports in other studies. Ventegodt ${ }^{20}$ found $0.9 \%$ gay and $1.2 \%$ bisexual among Danish sample aged $18-88$ years. Also in one of the cohorts used by Saewyc et $\mathrm{al}^{21}$, the British Columbia cohort, proportion of gay and bisexuals was found to be $0.5 \%$ and $2.1 \%$ respectively and Garofalo and colleagues ${ }^{22}$ found $2.5 \%$ of their adolescent sample self-identified as gay/lesbian or bisexual. Gay and bisexual respondents were more likely to be engaged in sex-related activity - dreams with sexual themes, masturbating, as well as oral and anal sex. This may suggest they are more sexually active, which may also account for the increased risk for HIV. It is possible that they may also be more willing to report sexual activity compared to heterosexual students. Males were more likely to be sexually active, had a lower age of sexual initiation and use protection during sex. This could stem from prevailing cultural norms of males being more sexual and imply an imbalance of power and issues about the right of women to initiate protection during sexual activity. There is also another matter of interest in our data which concerns the spread of HIV. It is known that one of the factors increasing vulnerability to HIV infections in same sex sexual relationships is anal sex and it should be of interest to the public health community that in our sample, $7.5 \%$ of respondents in heterosexual relationships still practiced anal sex. A popular reason among adolescents is that it prevents pregnancy but the implications for HIV and other STIs may be completely lost on them.

\section{Quality of life}

In our study, females reported higher overall quality of life. This may be because of the wide network of support from friends and family that women informally build up around themselves. The authors do not believe that this suggests a lack of conflict but an adaptive, maybe even resigned outlook that makes the average woman posit "it could be worse" and thus gives a sense of optimism in gauging their overall quality of life.

There is a direct relationship between the frequency of worship and quality of life. This may be because those who worship more frequently have a stronger social network and may be buffered against adverse events.

Those who self-identified as gay/bisexual had a lower average score on all domains of quality of life which was statistically significant on the physical health, psychological health and social relationships domains and this finding has been reiterated in other studies ${ }^{11,12}$. The reasons for this finding appear to be multiple. Studies have shown that GLB individuals are more vulnerable to HIV infections and other comorbidities ${ }^{23,24}$; stigma, homophobia, rife discrimination and outright criminalisation of same sex relationships could be other factors for this finding.

Feeling less accepted by the social environment, due to sexual minority status as well as the stigma associated with homosexuality, may also be associated with negative psychological consequences including reduced self-esteem and negative emotions including depression and anxiety ${ }^{11,25,26}$; especially in countries where the acceptance of homosexuality is low. All these factors may directly impair sexual life as well as emotional well-being $^{27,28}$ and may account for the lower quality of life found among the homosexual respondents in this study as has been described by Træen et al. ${ }^{12}$.

\section{Conclusion}

Same sex sexual attraction and behaviour are present among young people in Nigeria and this doubtlessly has serious implications for their sexual and reproductive health. This is especially important with the consistent finding of much lower levels of homosexuality compared to bisexuality, signaling a hidden socially unaccepted homosexual relationship and an open socially acceptable heterosexual relationship. In pushing the 
zero new infection of HIV, there are serious implications to consider.

There is some discordance between self-identified sexual orientation and sexual attraction/behaviour among the respondents. This discordance may be due in part to the criminalization of same sex relationships in the country, strict cultural norms and rife discrimination in the society against those who self-identify as LGB. More studies should be undertaken to explore these issues in planning interventions that would educate on safe sexual practices among this group. Also, asking about sexual attraction and behaviour may elicit more information than asking about sexual identity alone.

LGB youth report a lower QOL in certain domains compared to heterosexual counterparts and this may suggest some level of distress among these vulnerable group, thus, intervention programs and health services should target the unique needs of youths that self-identify as LGB.

\section{Limitations}

Multi-stage sampling technique used in this study has its limitations. Furthermore, this study was carried out among university students in Nigeria and the findings may not be generalizable to the general population.

\section{References}

1. Sturdevant M, Remafedi G. Special health care needs of homosexual youth. Adolescent Medicine. 1992;3:35971.

2. Pathela P, Blank S, Sell RL, Schillinger JA. The importance of both sexual behaviour and identity. American Journal of Public Health. 2006;96(5):765.

3. Sexual Minority Assessment Research Team (SMART). Best Practices for Asking Questions about Sexual Orientation on Surveys. The Williams Institute, 2009.

4. Sell RL. Defining and Measuring Sexual Orientation: A Review. Archives of Sexual Behaviour. 1997;26(6):64358.

5. Saewyc EM, Bauer GR, Skay CL, Bearinger LH, Resnick MD, Reis E, et al. Measuring sexual orientation in adolescent health surveys: Evaluation of eight school-based surveys. Journal of Adolescent Health. 2004;35(345):e1-e16.

6. Russell ST, Joyner K. Adolescent sexual orientation and suicide risk: evidence from a national study. American Journal of Public Health. 2001;91(8):1276-81.

7. Cochran SD, Mays VM. relation between psychiatric syndromes and behaviourally defined sexual orientation in a sample of the US population. American Journal of Epidemiology. 2000;151(5):516-23.

8. Saewyc EM, Homma Y, Skay CL, Bearinger L, Resnick M, Reis E. Protective factors in the lives of bisexual adolescents in North America. American Journal of Public Health. 2009;99:110-7.

9. Laumann EO, Gagnon JH, Michael RT, Michaels S. The social organization of sexuality: sexual practices in the United States. Chicago: University of Chicago Press; 1994.

10. Remafedi G, Resnick M, Blum R. Demography of sexual orientation in adolescents. Pediatrics. 1992;89:71421 PubMed.

11. Sandfort T, deGraaf R, Bijl RV. Same-Sex Sexuality and Quality of Life: Findings from the Netherlands Mental Health Survey and Incidence Study. Archives of Sexual Behaviour. 2003;32(1):15-22.

12. Træen BI, Martinussen M, Vitterso J, Saini S. Sexual orientation and quality of life among university students from Cuba, Norway, India and South Africa. Journal of Homosexuality. 2009;56(5):655-69.

13. VorugantiL, Heslegrave R, Awad AG, Seeman MV. Quality of life measurement in schizophrenia: reconciling the quest for subjectivity with the question of reliability. Psychological Medicine. 1998;28:165-72.

14. Bow-Thomas CC, Velligan DI, Miller AL, Olsen J. Predicting quality of life from symptomatology in schizophrenia at exacerbation and stabilization. Psychiatry Research. 1999;86(2):131-42.

15. Lehman AF. Instruments for measuring quality of life in mental illnesses. In: Katschnig H, Freeman H, Sartorius N, editors. Quality of life in mental disorders. New York: Wiley; 1997.

16. WHOQOL Group. The WHO Quality of Life Assessment (WHOQOL): Development and general psychometric properties. Social Science and Medicine. 1998;46:1569-85.

17. Lenth RV. Java applets for power and sample size2000 10/03/2015.

18. Araoye MO. Research methodology with statistics for health and social sciences. Ilorin: Nathadex; 2004.

19. Olusina AK, Ohaeri JU. Subjective qualilty of life of recently discharged Nigerian psychiatric patients. Social Psychiatry and Epidemiology. 2003;38:707-14.

20. Soren V. Sex and the quality of life in Denmark. Archives of Sexual Behaviour. 1998;27(3):295.

21. SaewycE, Skay C, Richens K, Reis E, Poon C, Murphy A. Sexual Orientation, Sexual Abuse, and HIV-Risk Behaviours among Adolescents in the Pacific Northwest. American Journal of Public Health. 2006;96(6). 22. Garofalo RP, Wolf RC, Kessel S, Palfrey J, Dagger 
RHD. The association between health risk behaviours and sexual orientation among a school-based sample of adolescents. Pediatrics. 1998;101(5):895 PubMed -902. 23. Centers for Disease Control (CDC). HIV/AIDS Surveillance Report. Atlanta Georgia: CDC, 1999.

24. Mustanski BS, Dupree MG, Bocklandt CM, Schork NJ, Hamer DH. A genomewide scan of male sexual orientation. Human Genetics. 2005;116(4):272 PubMed $-8$.

25. Leary MR. Making sense of self-esteem. Current Directions in Psychological Science. 1999;8(1):32-5.

26. Yanos PT, Rosenfield S, Horwitz AV. Negative and supportive social interactions and quality of life among persons diagnosed with severe mental illness. Community Mental Health Journal. 2001;37(5):405-19.

27. Muscatello MR, Bruno A, Scimeca G, Pandolfo G, Paduano R, Mico U, et al. The relationship between anger and heterosexual behavior. An investigation in a nonclinical sample of urban Italian undergraduates. The journal of sexual medicine. 2010;7(12):3899-908.

28. Scimeca G, Bruno A, Pandolfo G, Micò U, Romeo VM, Abenavoli E, et al. Alexithymia, Negative Emotions, and Sexual Behavior in Heterosexual University Students from Italy. Archives of Sexual Behaviour. 2013;42(1):117-27. 